\title{
Review of: "Axial Stress Provides a Lower Bound on Shear Wave Velocity in Active and Passive Muscle"
}

Xiao Liu

Potential competing interests: The author(s) declared that no potential competing interests exist.

This study investigated the relationship between muscle stress and SWV in passive and active muscle tissue. The study also established a stress model which fits passive data well, but only sets the lower bound for SWV measured in active muscle. The study design is appropriate, the aim and the results are well explained.

Several minor revisions may increase the quality of the paper:

1 There were many steps in the test process. It is suggested to add photos or illustrations to facilitate readers' understanding.

2 A three-line table was recommended for table 1. 